\title{
Acetylation of Microtubules Influences Their Sensitivity to Severing by Katanin in Neurons and Fibroblasts
}

\author{
Haruka Sudo and Peter W. Baas \\ Department of Neurobiology and Anatomy, Drexel University College of Medicine, Philadelphia, Pennsylvania 19129
}

Here we investigated whether the sensitivity of microtubules to severing by katanin is regulated by acetylation of the microtubules. During interphase, fibroblasts display long microtubules with discrete regions rich in acetylated tubulin. Overexpression of katanin for short periods of time produced breaks preferentially in these regions. In fibroblasts with experimentally enhanced or diminished microtubule acetylation, the sensitivity of the microtubules to severing by katanin was increased or decreased, respectively. In neurons, microtubules are notably more acetylated in axons than in dendrites. Experimental manipulation of microtubule acetylation in neurons yielded similar results on dendrites as observed on fibroblasts. However, under these experimental conditions, axonal microtubules were not appreciably altered with regard to their sensitivity to katanin. We hypothesized that this may be attributable to the effects of tau on the axonal microtubules, and this was validated by studies in which overexpression of tau caused microtubules in dendrites and fibroblasts to be more resistant to severing by katanin in a manner that was not dependent on the acetylation state of the microtubules. Interestingly, none of these various findings apply to spastin, because the severing of microtubules by spastin does not appear to be strongly influenced by either the acetylation state of the microtubules or tau. We conclude that sensitivity to microtubule severing by katanin is regulated by a balance of factors, including the acetylation state of the microtubules and the binding of tau to the microtubules. In the neuron, this contributes to regional differences in the microtubule arrays of axons and dendrites.

\section{Introduction}

Microtubule severing is a physiological process whereby severing proteins such as katanin and spastin break the lattice of the microtubule through ATP hydrolysis (Roll-Mecak and McNally, 2010). This results in higher numbers of microtubules and more free ends of microtubules to interact with various proteins and structures in the cell (Roll-Mecak and Vale, 2006). In addition, the shorter length of the microtubules renders them more readily transported and reorganized by molecular motor proteins (Baas et al., 2006). Cells generally express high amounts of the severing proteins, typically high enough to completely sever all cellular microtubules if the severing proteins had unfettered access to them (Solowska et al., 2008). This raises the question as to how the severing activities of katanin and spastin are regulated, and how they are targeted to particular microtubules or particular regions on microtubules.

Microtubule severing is generally considered to impact more stable microtubules. An attractive possibility, in this regard, is that the severing proteins are targeted to microtubules that are rich in posttranslational modifications that accompany stability. Indeed, there is a growing body of evidence suggesting that vari-

Received Jan. 5, 2010; revised April 1, 2010; accepted April 7, 2010.

This work was funded by grants from the National Institutes of Health, National Science Foundation, and Alzheimer's Association (P.W.B.). Tubacin was kindly provided by Dr. Stuart L. Schreiber of Harvard University. We thank Dr. Carsten Janke of Centre National de la Recherche Scientifique and Dr. Kristen Verhey of the University of Michigan for advice and helpful discussions.

Correspondence should be addressed to Peter W. Baas, Department of Neurobiology and Anatomy, Drexel University College of Medicine, 2900 Queen Lane, Philadelphia, PA 19129. E-mail: pbaas@drexelmed.edu.

DOI:10.1523/JNEUROSCI.0048-10.2010

Copyright $\odot 2010$ the authors $\quad 0270-6474 / 10 / 307215-12 \$ 15.00 / 0$ ous microtubule-related proteins have differential affinities for microtubules rich or deficient in tubulin subunits that bear modifications such as acetylation, detyrosination, or polyglutamylation (Hammond et al., 2008). For example, recent studies suggest that acetylation may play an important role in regulating the degree to which kinesin- 1 interacts with the microtubules (Reed et al., 2006).

Acetylation occurs on $\alpha$-tubulin and is unique among the various modifications in that it occurs on the luminal face of the microtubule (for review, see Hammond et al., 2008). It occurs on lysine 40 , which is deeply embedded within the tubulin subunit. Thus, compared with the other modifications, it is less clear how acetylation could affect the properties of the microtubule or the manner by which it interacts with other proteins. In addition, it is unclear how the enzymes that add or remove the acetyl group access the relevant site. Two different enzymes have been identified that can remove the acetyl group, namely HDAC6 and Sirt2. Depletion of either enzyme results in markedly enhanced acetylation of microtubules, suggesting that the two enzymes may function coordinately. A variety of studies over the years indicate that acetylation is the result of stabilization and not the cause of it, although one somewhat controversial study suggests otherwise (Matsuyama et al., 2002).

In neurons, microtubule severing is critically important for the development of axons and dendrites and is particularly robust at sites of impending branch formation (Yu et al., 2005, 2008; Riano et al., 2009). It is known that microtubules in axons are generally richer in acetylated tubulin than are dendritic microtubules, that acetylated tubulin is unevenly distributed along the length of the microtubules, and that microtubules vary in their 
levels of acetylated tubulin in different regions of the axon (Cambray-Deakin and Burgoyne, 1987; Baas et al., 1991). For these reasons, acetylation makes particularly good sense in the neuron as a potential means to earmark certain microtubules for severing.

\section{Materials and Methods}

DNA constructs. Constructs used for these studies were the following: pEGFP-C1 (control construct in experiments for neuron; BD Biosciences), pEGFP-C1-p60 [C-terminally enhanced green fluorescent protein (EGFP)-tagged rat p60-katanin] (Qiang et al., 2006), pEGFPC1-M85 (C- terminally EGFP-tagged mouse spastin translated from the second start codon), pcDNA human HDAC6 flag (ID number 13823) (addgene), and pRC/cytomegalovirus (CMV) promoter-Flaghtau441wt (flag-tagged human four-repeat tau; provided by Dr. R. Brandt, University of Osnabrück, Barbarastrasse, Germany).

Pharmacologic tools. Trichostatin A (TSA) was purchased Millipore Corporation. Taxol was purchased from Sigma. Tubacin was provided by Dr. Stuart L. Schreiber (Harvard University, Cambridge, MA).

Cell culture, transfection, and drug treatments. Rat RFL-6 fibroblasts were cultured as described previously (Qiang et al., 2006) and transfected with pEGFP-C1-p60, pEGFP-C1-M85, pHDAC6-flag, or pRC/CMVFlaghtau441wt using a Nucleofector (Amaxa) with program G-13 from the manufacturer. Fifteen micrograms of each plasmid and $10^{6}$ cells were used for each transfection. The cells were plated at a density of 4000 cells per well on glass coverslips mounted in the bottom of 35-mm-diameter Petri dishes with holes drilled in the bottom. Taxol, tubacin, and TSA were used at $5 \mu \mathrm{M}, 10 \mu \mathrm{M}$, and $300 \mathrm{nM}$, respectively. Drugs were added to the culture immediately after transfection. Cells were cultured for $24 \mathrm{~h}$ (experiments in Fig. 1-3, 6) or $12 \mathrm{~h}$ (experiments in Fig. 4) after transfection before being fixed.

Hippocampal neurons. Rat hippocampal neurons were prepared as described previously (Yu and Baas, 1994), except that they were cultured in the presence of conditioned media obtained from primary cultured astroglial cells derived from neonatal rat whole brain. The conditioned medium is a filtered supernatant of culture media of astroglial cells that had been cultured for $2 \mathrm{~d}$ under the hippocampal neuron plating medium (Neurobasal medium supplemented with 2\% B27, 0.3\% glucose, 1 mu glutamine, and 5\% FBS). The cells were plated at a density of 3000 cells per well on glass coverslips mounted onto the bottom of 35- mmdiameter Petri dishes with holes drilled in the bottom. The transfections of pEGFP-C1-p60, pEGFP-C1-M85, pHDAC6-flag, or pRC/CMVFlaghtau441wt were performed $8 \mathrm{~d}$ after the plating. For transfections of those constructs, we used Lipofectamine 2000 (Invitrogen). Two micrograms of DNA constructs and $5 \mu$ l of Lipofectamine 2000 were used per $35 \mathrm{~mm}$ dish. The cells were incubated in the DNA/Lipofectaminecontaining medium for $5 \mathrm{~h}$. Then neurons were transferred to fresh $37^{\circ} \mathrm{C}$ hippocampal neuron plating medium. In experiments using drugs, the drug was added to the medium at this time. Twenty-four hours later, cells were fixed for immunostaining. Transfection efficiency was generally 5-10\% for neuronal cultures and $15-30 \%$ for RFL- 6 cell cultures.

Immunofluorescence techniques. Cyanine 3 (Cy3)-conjugated monoclonal anti- $\beta$-tubulin (1:150; for general tubulin staining), monoclonal anti-acetylated tubulin (6-11B-1) (1:400), monoclonal anti-flag (M2) (1:500), and monoclonal anti-GFP (1:250; for the enhancement of GFP signals in experiments of neuron) antibodies were purchased from Sigma. Rabbit polyclonal anti-GFP antibody (1:250; for the enhancement of GFP signals in experiment of Fig. 4) was purchased from Abcam. Rabbit polyclonal anti-human HDAC6 (H-300) (1:200) was purchased from Santa Cruz Biotechnology. Monoclonal anti-polyglutamylated tubulin antibody (GT335) (1:500) was provided by Dr. Carsten Janke (Centre National de la Recherche Scientifique, Montpellier, France). Monoclonal anti-detyrosinated tubulin antibody (4B8) (1:300) was provided by Dr. G. G. Gundersen (Columbia University, New York, NY). Monoclonal anti-tau antibody (Tau-1), used for dephosphorylated tau staining (1:500), was purchased from Sigma.

For immunofluorescence studies, cultures were briefly washed with $37^{\circ} \mathrm{C} \mathrm{PBS}$ and then simultaneously fixed and extracted with $4 \%$ parafor- maldehyde, $0.2 \%$ glutaraldehyde, and $0.1 \%$ Triton X-100 for 15 min. Cultures were washed with PBS three times for $5 \mathrm{~min}$, quenched with 2 $\mathrm{mg} / \mathrm{ml}$ sodium borohydride three times for $10 \mathrm{~min}$, and then blocked with $10 \%$ normal goat serum and $10 \mathrm{mg} / \mathrm{ml} \mathrm{BSA}$ in PBS for $1 \mathrm{~h}$. For immunostaining of the expressed tau, HDAC6, and/or severing proteins, cultures were prepared as described previously (Karabay et al., 2004). In experiments using RFL-6 except for that of Figure 4, we detected the GFP signals of tagged-severing proteins without additional enhancement, whereas in experiments using neurons or that of Figure 4, we detected the GFP signals of tagged-severing proteins with enhancement by staining with monoclonal (for neurons) or polyclonal (for Fig. 4) anti-GFP antibody. We detected expressed HDAC6 with anti-human HDAC6 antibody, which could reveal the presence of exogenous human HDAC6 in rat-derived fibroblasts or neurons.

We detected expressed human tau protein by staining with Tau-1 antibody in both RFL- 6 cells and neurons when they were costained with anti-acetylated tubulin antibody. In RFL- 6 cells, we could clearly identify highly exogenous tau-expressing cells because they do not express endogenous tau. Although neurons have endogenous tau, we could identify transfected cells with much higher expression levels of tau, especially in their dendrites. In exogenous tau-expressing RFL- 6 cells and neurons, we identified morphological changes in general tubulin staining, namely microtubule bundle formation in RFL- 6 cells and the generation of long straight microtubules crossing in all directions in the cell bodies of neurons. The tau expression by itself in neurons caused the reduction of microtubule levels in cell bodies. We identified tau-expressing RFL-6 cells and neurons based on those morphological characteristics in the following experiments in which we tested the microtubule sensitivities to exogenous katanin.

To quantify microtubule mass, both fibroblasts and neurons were simultaneously fixed and extracted to remove free tubulin as described previously (Yu et al., 2005) and then immunostained for HDAC6, tau (in the case of Fig. 6), and GFP (in the case of neurons and Fig. 4). Cultures were then treated with appropriate fluorescence-conjugated secondary antibodies and subsequently with Cy3-conjugated anti- $\beta$-tubulin antibody as third antibody. So that samples could be compared against one another, microscope settings were adjusted to equilibrate the signal. In experiments on cultured neurons, we selected only neurons that were sufficiently separated from neighboring cells that we could distinguish axons from dendrites on the basis of morphology. Images were acquired with an AxioVert 200M microscope (Carl Zeiss) coupled with an Orca-ER Digital CCD (Hamamatsu) and a 100× Plan-Neorofluar/1.3 numerical aperture objective. Except for the GFP signal quantification (see below), images to be compared were taken at identical settings of exposure time, brightness, and contrast and analyzed with Axiovision 4.0 software. Measurements were taken as total fluorescence intensity per cell in the case of RFL- 6 cells and total fluorescence intensity in $10 \mu \mathrm{m}$ regions of axons or dendrites. In the latter case, we made sure to include the region of strongest fluorescence within any given axon or dendrite. Statistics were done using Student's $t$ test. In RFL-6 experiments, we classified GFP-fusion protein-expressing cells according to their total GFP signal intensity per cell. We set exposure times at 150 and $750 \mathrm{~ms}$ for GFP-katanin and GFP-spastin detection, respectively. Other settings, including brightness and contrast, are identical throughout the experiments. We defined cells possessing background-subtracted GFP fluorescence intensity from 0 to 300 and 0 to 400 arbitrary fluorescence units (AFU) as low expressors, from 300 to $800 \mathrm{AFU}$ and from 400 to 700 as medium expressors, and from 800 to 2000 and from 700 to 2000 as high expressors, for GFP-katanin and GFP-spastin expressing cells, respectively.

\section{Results}

For the present studies, as with our previous work on the microtubule-severing proteins (Qiang et al., 2006, 2010; Yu et al., 2008), we used RFL-6 rat fibroblasts and cultured rat hippocampal neurons. The fibroblasts are useful for high-resolution imaging of microtubules because they are so flat. In addition, they are useful for certain studies because they do not endogenously ex- 
Control

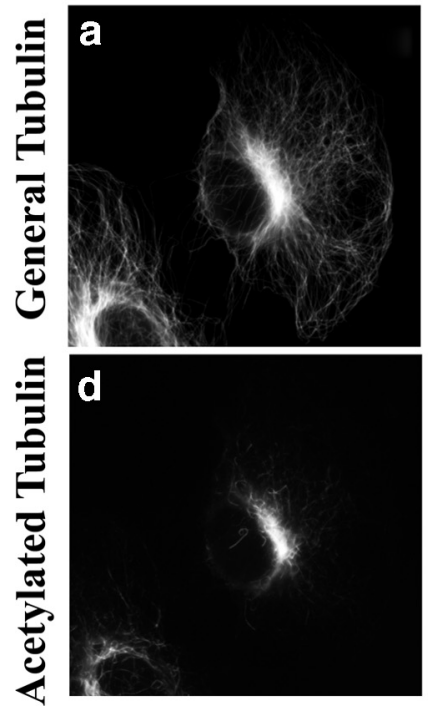

HDAC6

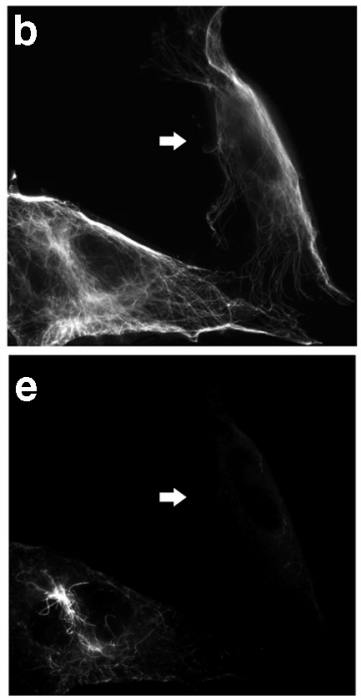

Tubacin

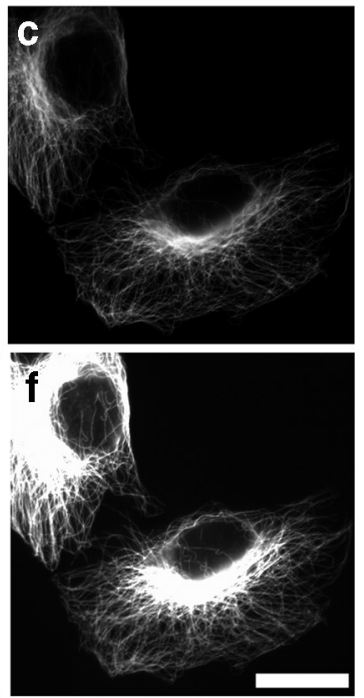

Figure 1. Effects of HDAC6 overexpression and tubacin treatment on cellular microtubule acetylation in RFL- 6 cells. Top row shows general tubulin staining, and bottom row shows acetylated tubulin staining of the same cells. $\boldsymbol{d}$-f, Compared with control (d), HDAC6-overexpressing cells (arrow) show less microtubule acetylation (e) and tubacin-treated cells show enhanced microtubule acetylation $(\boldsymbol{f}) \cdot \boldsymbol{a}-\boldsymbol{c}$, The microtubule arrays of HDAC6-0verexpressing cells $(\boldsymbol{b})$ or tubacin-treated cells $(\boldsymbol{c})$ show no obvious changes in organization compared with controls $(\boldsymbol{a})$. Scale bar, $20 \mu \mathrm{m}$.

press microtubule-associated proteins (MAPs) such as tau that could impact the sensitivity of the microtubules to severing. The hippocampal neurons are useful because they generate bona fide and morphologically distinguishable axons and dendrites.

\section{Microtubules rich in acetylated tubulin are preferentially severed by katanin but not spastin}

In a previous study, we found that treatment of RFL- 6 rat fibroblasts with taxol does not alter the diminution of microtubule mass observed with katanin overexpression, despite the fact that taxol stabilizes and bundles microtubules (Qiang et al., 2006). This was surprising because, even if taxol has no effect on sensitivity to katanin, we would have thought that the greater stability of the microtubules in the presence of taxol should reduce the ensuing depolymerization of microtubule mass that occurs as a result of the severing. On this basis, we became suspicious that taxol treatment might actually render the microtubules more sensitive to severing by katanin. Given that taxol causes a rapid accumulation of tubulin posttranslational modifications (Gundersen et al., 1987; Piperno et al., 1987), we hypothesized that it might be one or more of these modifications that renders the microtubules more sensitive to severing by katanin. To investigate this further, we used previously characterized tools for the study of microtubule acetylation in living cells. HDAC6, a histone deacetylase that is specific for tubulin, has been reported to cause global deacetylation of microtubules when overexpressed in cells (Hubbert et al., 2002; Zhao et al., 2010). TSA is a membrane-permeable drug that inhibits a broad range of deacetylases and hence causes microtubules in cells to become highly acetylated (Matsuyama et al., 2002; Chang et al., 2009; Zilberman et al., 2009). Tubacin, a more recently developed membrane-permeable drug, is preferable for these studies because it specifically inhibits HDAC6 and hence elevates tubulin acetylation without affecting other cellular proteins that can be acetylated (Haggarty et al., 2003; Chang et al., 2009; Zilberman et al., 2009).
In a first set of experiments, we confirmed the efficacy of these tools in RFL-6 fibroblasts. In some cases, cultures were transfected to overexpress HDAC6 (using a human HDAC6 construct with a flag tag; see Materials and Methods) and then fixed $24 \mathrm{~h}$ later. Other cultures were treated with $300 \mathrm{~nm}$ TSA, $10 \mu \mathrm{M}$ tubacin, or $0.1 \%$ DMSO (vehicle alone, control) for $24 \mathrm{~h}$ and then fixed. The fixed cultures were then prepared for multichannel immunofluorescence visualization of general tubulin and acetylated tubulin. In the case of the HDAC6 overexpression studies, cultures were also immunolabeled for human HDAC6. Details on immunofluorescence procedures and antibodies are provided in Materials and Methods. Representative images are shown in Figure 1. Figure $1 a-c$ shows staining for general tubulin, and the bottom panels (Fig. $1 d-f$ ) show staining for acetylated tubulin in the same cells. Control cells displayed a broad splayed array of microtubules revealed with the antibody for general tubulin and a center-focused subarray of curved microtubules revealed by the antibody for acetylated tubulin (Fig. 1a,d). This is the typical pattern for general and acetylated tubulin observed in most tissue culture fibroblasts (Piperno et al., 1987). In HDAC6overexpressing cells, we observed notable reduction in staining for acetylated tubulin. The morphology of the cells appeared slightly more elongated than controls and the microtubules appeared slightly less splayed (Fig. $1 b, e$ ). It was necessary to select the most highly overexpressing cells to visualize the notable reduction in acetylation, so from this point forward, for all studies reported here with HDAC6, we chose high expressors. In tubacin-treated cultures, all cells displayed uniformly and dramatically stronger staining for acetylated tubulin, but there were no recognizable differences in morphology or general tubulin staining relative to controls cells (Fig. 1c,f). TSA treatment yielded results similar to tubacin treatment (data not shown). The effects of tubacin, TSA, and HDAC6 were confirmed in three independent experiments for each condition. These results are consistent with those of previous reports (Hubbert et al., 2002; Haggarty et al., 2003). Quantitative analysis (see Fig. 3k) showed that there are slight increases in total microtubule levels in tubacin- and TSA-treated cells, respectively, but no such difference in HDAC6-expressing cells compared with controls.

Taxol treatment is known to cause microtubules in cells to become highly acetylated, detyrosinated, and polyglutamylated (Gundersen et al., 1987; Piperno et al., 1987). We wanted to ascertain whether taxol might impact microtubule severing by katanin differently if we specifically inhibit its capacity to enhance microtubule acetylation. For this, we introduced taxol into the cultures simultaneously with the HDAC6 transfection. As shown in Figure $2 A$, this experimental regimen was effective at severely diminishing the levels of microtubule acetylation with no diminution in the levels of the other two modifications. In addition, there was no apparent effect on the levels or bundling of the microtubules as a result of diminished acetylation during taxol treatment. These results are important because, in some experimental regimens, there seems to be a link among the modifica- 
A
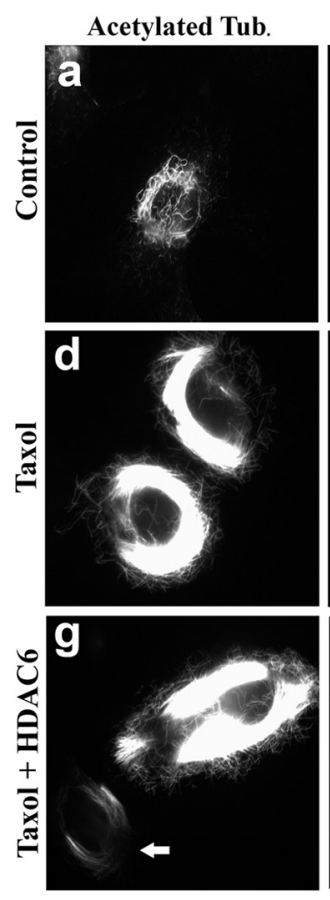

Detyrosinated Tub.
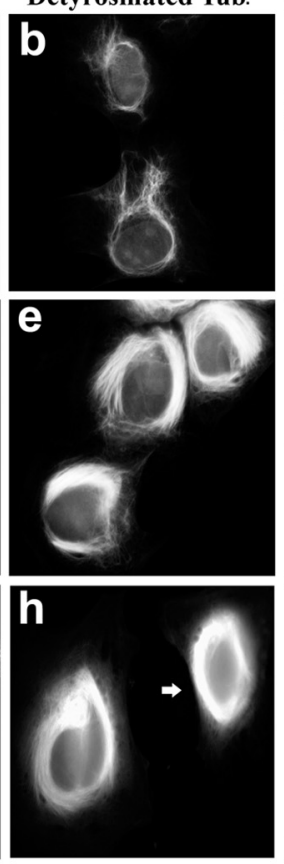

B
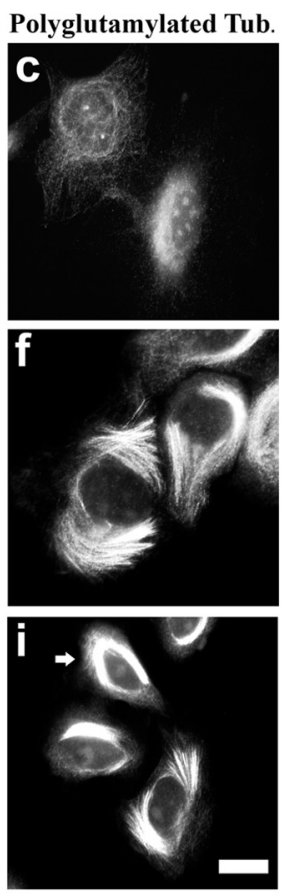
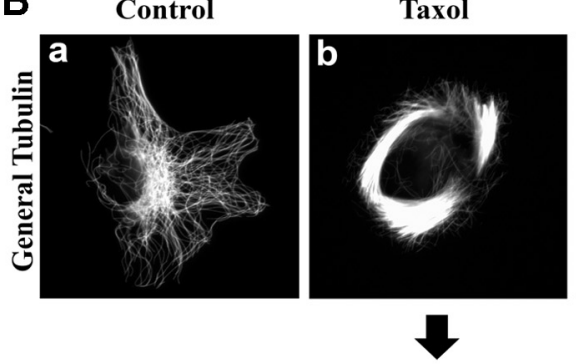

Taxol + HDAC6

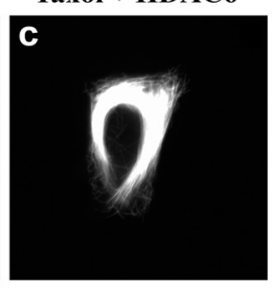

Middle level GFP-P60 Katanin expression
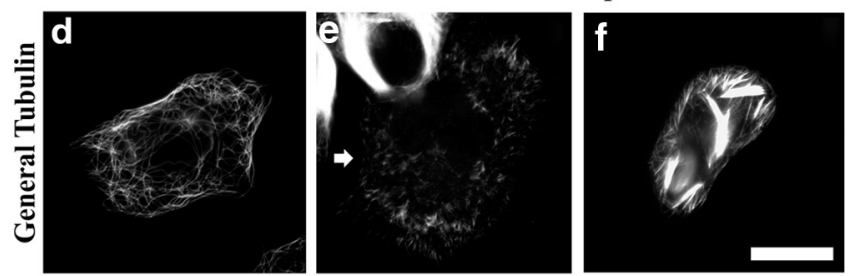

g

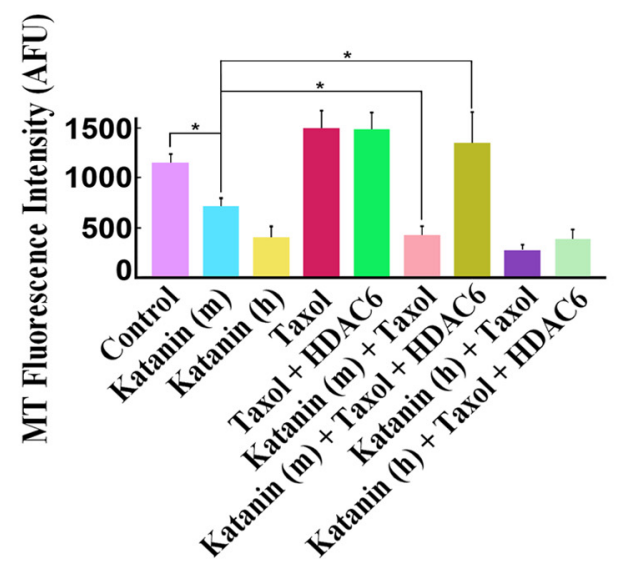

Figure 2. Effects of taxol on katanin-mediated microtubule-severing. $\boldsymbol{A}$, Cells were immunostained for acetylated tubulin $(\boldsymbol{a}, \boldsymbol{d}, \boldsymbol{g})$, detyrosinated tubulin $(\boldsymbol{b}, \boldsymbol{e}, \boldsymbol{h})$, or polyglutamylated tubulin $(\boldsymbol{c}, \boldsymbol{f}, \boldsymbol{i})$. Top row shows controls $(\boldsymbol{a}-\boldsymbol{c})$, middle row shows taxol-treated cells $(\boldsymbol{d}-\boldsymbol{f})$, and bottom row shows cells treated with taxol + HDAC6 (arrows in $\boldsymbol{g}-\boldsymbol{i})$. Compared with control $(\boldsymbol{a}-\boldsymbol{c})$, all three modifications were enhanced in taxol-treated cells $(\boldsymbol{d}-\boldsymbol{f})$. HDAC6 overexpression specifically reduced microtubule acetylation in taxol-treated cells (arrow in $\boldsymbol{g}$ ) but not the other modifications (arrows in $\boldsymbol{h}, \boldsymbol{i}) \cdot \boldsymbol{B}, \mathrm{RFL}-6$ cells were treated with taxol or taxol + HDAC6 and tested for their microtubule sensitivity to middle-level katanin overexpression. Top row shows microtubules in controls $(\boldsymbol{a})$, taxol-treated cells $(\boldsymbol{b})$, and taxol + HDAC6-treated cells $(\boldsymbol{c})$. Bottom row $(\boldsymbol{d}-\boldsymbol{f})$ shows the microtubules of katanin overexpressors under the conditions of corresponding top row. All images show general tubulin staining. Taxol-treated cells show robust bundle formation $(\boldsymbol{b}, \boldsymbol{c})$, regardless of HDAC6 overexpression. Compared with controls (d), taxol-treated cells show enhanced microtubulesevering $(\boldsymbol{e})$, but the enhancement is inhibited by HDAC6 overexpression $(\boldsymbol{f})$. Scale bar, $20 \mu \mathrm{m} . \boldsymbol{g}$ shows quantification of microtubule (MT) levels by general tubulin staining intensity. Katanin (h) and Katanin $(\mathrm{m})$ indicate high-level and middle-level katanin overexpression, respectively. ${ }^{*} p<0.01$ (Student's $t$ test).

tions such that affecting one can affect the others (Redeker et al., 2005; Ikegami et al., 2007). Figure $2 B a-B c$ further confirms that taxol treatment results in dense microtubule bundles whether or not HDAC6 is overexpressed. The increase in total microtubule mass as a result of taxol treatment was $30 \%$ higher than control cells, whereas the increase in total acetylated microtubule mass was $616 \%$ higher than control cells (data not shown), indicating that the increased immunoreactivity for acetylated microtubule is indeed attributable to increased acetylation of microtubules, not just increased microtubule levels. High overexpression of katanin (rat GFP-p60-katanin) (Karabay et al., 2004; Yu et al., 2005, 2008; Qiang et al., 2006) resulted in 85\% loss of microtubule mass in control cells, 83\% loss in taxol-treated cells, and 75\% loss in cells treated with taxol during HDAC6 overexpression (Fig. $2 \mathrm{Bg}$ ). However, if we analyzed the medium expressors, we observed only $45 \%$ loss of microtubule mass in control cells and $73 \%$ loss in taxol-treated cells but no significant loss $(p>0.05)$ of microtubule mass if HDAC6 overexpression was included with the taxol treatment (with significant difference between katanin alone and katanin $+\operatorname{taxol}(p<0.01 ; n=3,25$ cells were analyzed for each condition) (Fig. 2Bd-Bg). These data support the hypothesis that the acetylation state of the microtubules is a critical factor in determining the sensitivity of the microtubules to being severed by katanin (although very high levels of katanin can apparently override this effect).

To further test the hypothesis, we conducted additional experiments without taxol but including the drugs that enhance microtubule acetylation. In addition, we included in these studies spastin, the other microtubule-severing protein that has been studied extensively in neuronal and non-neuronal cells (Zhang et al., 2007; Solowska et al., 2008). Again, cells were sorted with regard to levels of katanin or spastin expression, with medium expressors quantified separately from high expressors. Representative images are shown in Figure $3 a-j$, and the quantitative data on microtubule levels are shown in Figure $3 k$. In medium expressors of katanin ( $40 \%$ loss compared with control) but not spastin (57\% loss), we recognized significant downregulation or upregulation of microtubule severing in respective manipulations (Fig. $3 c-e, h-j)$. As shown by the data quantification (Fig. $3 k)(n=3,25$ cells were analyzed for each condition), the effects in both direc- 


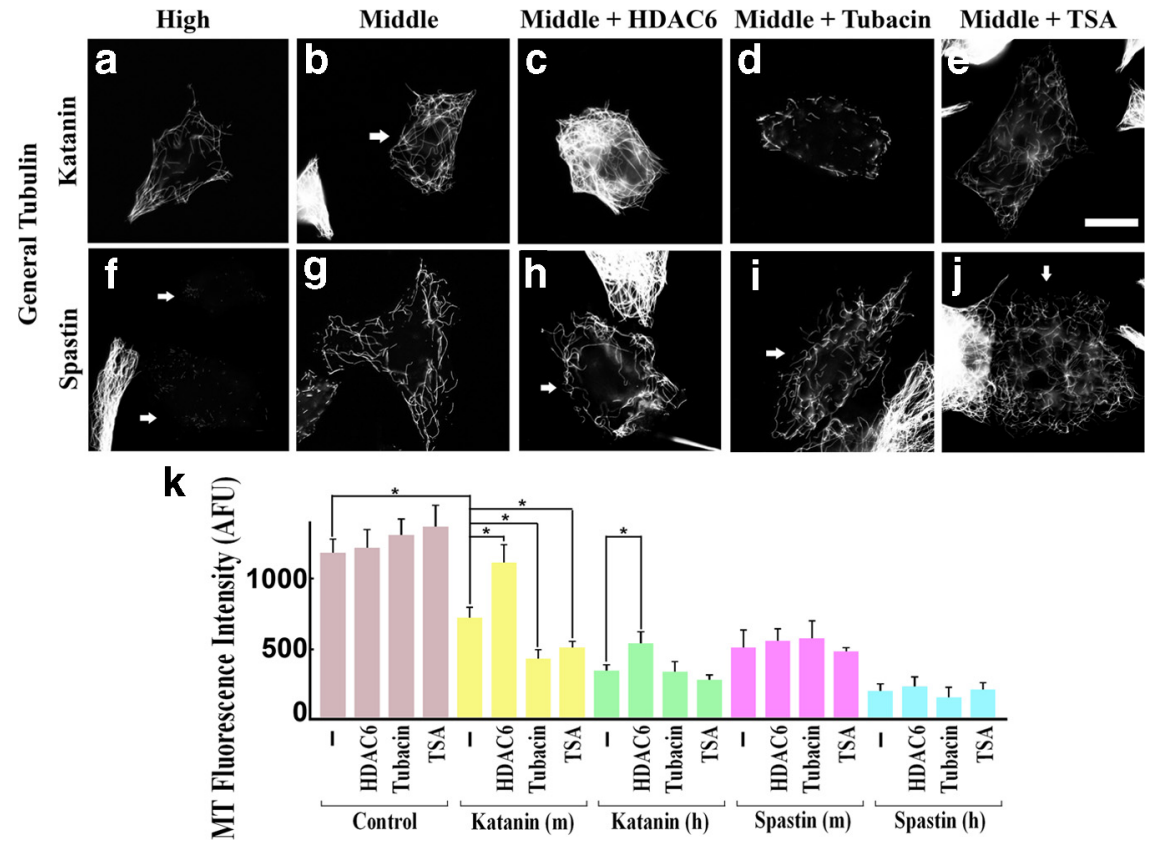

Figure 3. Effects of manipulations of microtubule acetylation on katanin/spastin-mediated microtubule severing. RFL-6 cells were transfected with katanin or spastin plasmids under conditions of HDAC6 overexpression or tubacin treatment. Top row $(\boldsymbol{a}-\boldsymbol{e})$ shows microtubules of katanin overexpressors revealed by general tubulin staining, and bottom row $(\boldsymbol{f}-\boldsymbol{j})$ shows those of spastin overexpressors. High, High-level katanin or spastin overexpressors; Middle, middle-level katanin or spastin overexpressors; Middle + HDAC6, middle-level katanin/spastin overexpressors with HDAC6 coexpression; Middle + Tubacin, middle-level katanin/spastin overexpressors under tubacin treatment; Middle + TSA, middle-level katanin/spastin overexpressors under TSA treatment. In middle-level katanin overexpressors, HDAC6 inhibited severing, whereas tubacin or TSA enhanced severing. Conversely, such effects on severing were not found in spastin overexpressors. Scale bar, $20 \mu \mathrm{m}$. $\boldsymbol{k}$, Quantification of microtubule (MT) levels by general tubulin staining intensity. ${ }^{*} p<0.01$ (Student's $t$ test).

tions were statistically significant especially in middle-level expressors. We observed 29 and $24 \%(p<0.01)$ significantly more severing in tubacin- and TSA-treated cells, respectively, but $31 \%$ $(p<0.01)$ significantly less severing in HDAC6-expressing cells. We should note that, although HDAC6 and katanin coexpressors showed protection of total microtubules from severing, they also showed a tendency of loss of their convergence in microtubule array to cell center, suggesting potential involvement of previously reported HDAC6-mediated primary cilia absorption mechanisms in this phenomenon (Pugacheva et al., 2007). Together, these results support the conclusion from the taxol studies that microtubules rich in acetylated tubulin are favored for severing by katanin but also indicate that the same is not true of spastin.

\section{Katanin-induced breaks are preferentially localized on acetylated microtubules}

We next wanted to pursue the conclusion from the above studies without the use of experimental manipulation of microtubule acetylation. If the conclusion from the above experiments is correct, we would surmise that katanin should preferentially cut the microtubules in regions normally rich in acetylated tubulin compared with regions deficient in acetylated tubulin, whereas spastin should have no such preference. When the severing proteins are overexpressed in cells, particularly for somewhat shorter periods of time, microtubules that had been in the process of being severed at the time of fixation can be readily visualized (Yu et al., 2008). So, to pursue this, we fixed the cells after only $12 \mathrm{~h}$ of katanin or spastin overexpression and then stained them for general tubulin and acetylated tubulin with enhancement of GFP signals by rabbit polyclonal anti-GFP antibodies, followed by FITC-conjugated anti-rabbit IgG as second antibody. For these studies, it was also helpful to focus on low expressors (in the presence of enhancement) so that we could more optimally discern microtubules in the process of being severed, and it was helpful to focus our analyses on the more central region of the cells in which acetylated regions of microtubules are most abundant. Breaks were identified with the general tubulin staining, and then, for each identified break, an image was obtained for acetylated tubulin. Each break was then scored as positive if a detectable signal for acetylated tubulin was observed within $1 \mu \mathrm{m}$ of the break on both sides of the break and scored as negative if this was not the case (Fig. 4). Percentages were calculated per each of experiments, and then a mean percentage for each severing protein was calculated. Using this procedure, we found that $62 \%$ of the breaks observed with katanin were in acetylated regions of the microtubules (for two different examples, see Fig. 4a, $b$ $, d, e, g, h)$, whereas only $24 \%$ of breaks observed with spastin were in acetylated regions (for a typical break in a microtubule region not rich in acetylated tubulin, see Fig. $4 c, f, i)$. Three independent experiments were performed for each of conditions, and $>30$ total breaks from $>30$ expressing cells were chosen for the analysis in each of experiments (katanin, $62 \pm 4.3 \%$; spastin, $24 \pm 5.3 \%$; there is a significant difference, $p<0.01$ ). To understand the meaning of these observations, we also calculated the percentage of randomly selected spots on microtubules that turned out to be flanked with acetylated regions. Microtubules were chosen in the same area of the control cells as in the case of the katanin- or spastin-overexpressing cells. The percentage of these spots that turned out to be flanked by acetylated regions was $20.0 \pm 3.4 \%$, which is only slightly lower than the percentage of breaks that occurred in acetylated regions when spastin was overexpressed but markedly lower than the percentage of breaks that occurred in acetylated regions when katanin was overexpressed. These observations support the conclusion that katanin, but not spastin, has a strong preference for severing microtubule regions rich in acetylated tubulin.

\section{Neurons display regional differences in the effects of} microtubule acetylation on sensitivity to katanin

The results on RFL-6 fibroblasts indicate that acetylation can be a critical factor in the sensitivity of cellular microtubules to severing by katanin, but the question remains as to whether this is a major regulatory mechanism in more complex cell types. Our next step was to test whether acetylation influences sensitivity of microtubules to katanin in neurons. Typical vertebrate neurons simultaneously maintain two different kinds of processes: a single axon and multiple dendrites. Microtubules in the axon are known to be much richer in acetylated tubulin than microtubules in dendrites (Cambray-Deakin and Burgoyne, 1987; Baas et al., 
1991), so acetylation makes sense as a potential means to locally differentiate microtubule severing in each compartment. We reported previously that $12 \mathrm{~h}$ of katanin overexpression results in substantial loss of microtubule mass from the cell body and dendrites of cultured rat hippocampal neurons but not from the axon (Yu et al., 2005). On the surface, this would appear to be the opposite of what would be expected on the basis of the results in fibroblasts described above, given that axonal microtubules are more highly acetylated. To investigate further, we used the same tools to manipulate acetylation as we used on the experiments with fibroblasts.

For these studies, we used rat hippocampal neurons that had been grown in culture for $9 \mathrm{~d}$, by which time axonal arbors were extensive and bona fide dendrites had formed. Tubacin, TSA, or DMSO alone was included in the media during the last $24 \mathrm{~h}$ of culture. Transfection with HDAC6 was conducted (using Lipofectamine for transfection; see Materials and Methods) during the final day of culture as well. Treatment with tubacin or TSA had no obvious effect on either the morphology of the neurons or amount of microtubule, and the same was true of HDAC6 overexpression (Fig. $5 a-c$ ). Dendritic microtubules showed similar responses to our manipulations (Fig. $5 e, f, k$ ) as that of fibroblasts, whereas axonal microtubules did not (Fig. $5 e, f, j$ ). Conversely, neurons overexpressing spastin displayed no difference in the sensitivity of the microtubules in axons compared with dendrites, as we reported previously (Yu et al., 2008). Similarly, no changes in response to the acetylation manipulations were found (Fig. $5 g-k$ ). Quantitatively, the amount of axonal microtubule in respective conditions are as follows: control $(2790 \pm 231)$, HDAC6 (2630 \pm 379$)$, tubacin $(2843 \pm 175)$, katanin (2509 \pm 262), katanin + HDAC6 (2559 \pm 238$)$, katanin + tubacin $(1932 \pm 162)$, spastin $(1769 \pm 264)$, spastin + HDAC6 (1608 \pm $239)$, and spastin + tubacin $(1678 \pm 305)$ as indicated by [mean microtubule mass $(\mathrm{AFU}) \pm \mathrm{SD}$ ] formula $(n=3,25$ cells were analyzed for each condition). Dendritic microtubules in respective conditions are as follows: control $(2387 \pm 404)$, HDAC6 (2405 \pm 416$)$, tubacin $(2268 \pm 307)$, katanin (1574 \pm $217)$, katanin + HDAC6 $(2480 \pm 390)$, katanin + tubacin $(891 \pm$ 298), spastin (1401 \pm 320$)$, spastin + HDAC6 (1333 \pm 356$)$, and spastin + tubacin $(1513 \pm 471)$. There are significant differences between katanin-expressing dendrites and katanin + HDAC6 dendrites or between katanin-expressing dendrites and katanin + tubacin dendrites $(p<0.01)$, although we than the control.
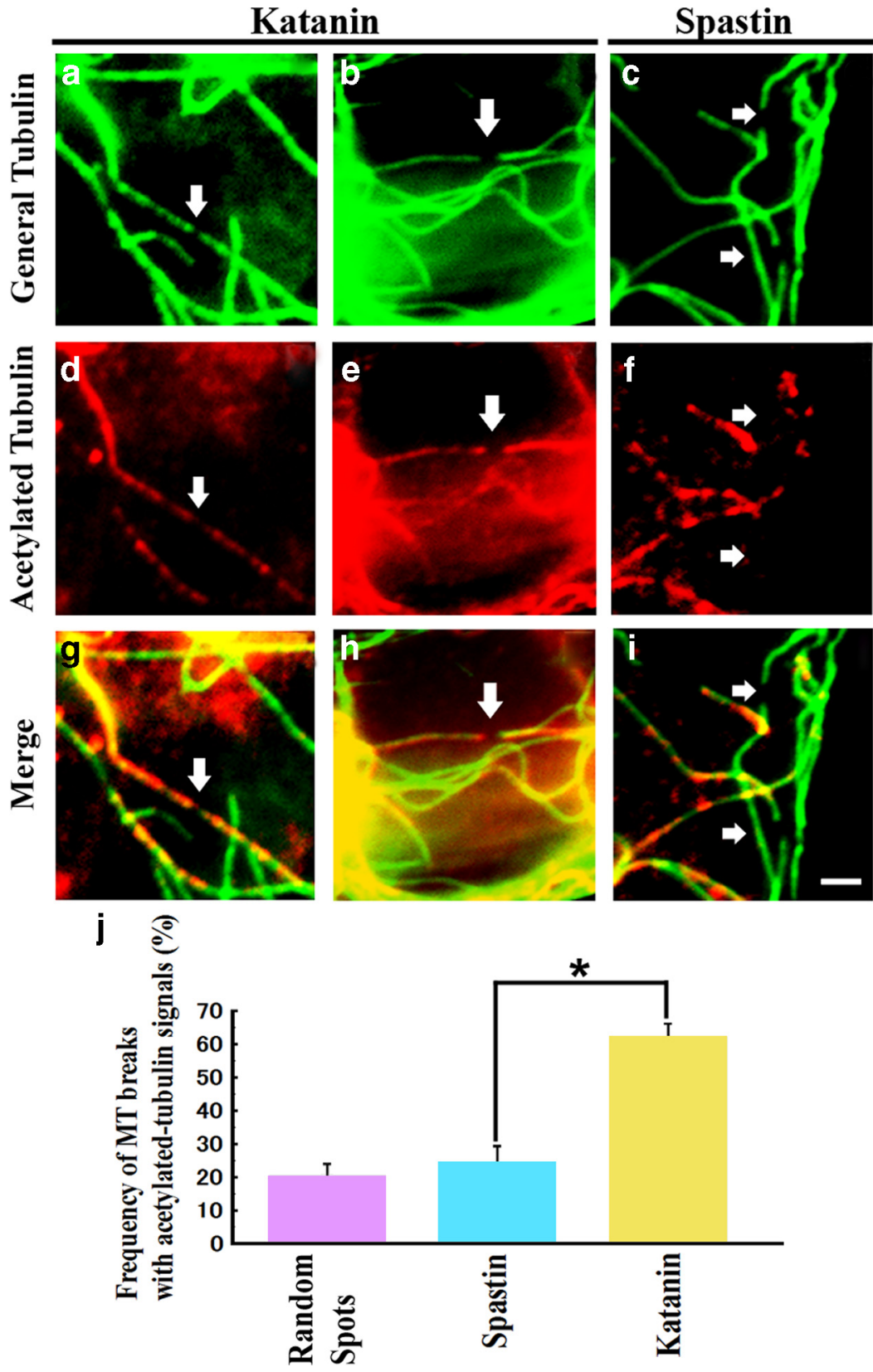

Figure 4. Microtubule breakpoint analysis. RFL-6 cells were transfected with katanin or spastin plasmids. Cells were fixed and coimmunostained for general tubulin and acetylated tubulin. Cells with low overexpression levels and discernable breakpoints were selected for analysis. Left and middle columns $(\boldsymbol{a}, \boldsymbol{b}, \boldsymbol{d}, \boldsymbol{e}, \boldsymbol{g}, \boldsymbol{h})$ show katanin overexpressors, and the right column $(\boldsymbol{c}, \boldsymbol{f}, \boldsymbol{i})$ show spastin overexpressor. Top row $(\boldsymbol{a}-\boldsymbol{c})$ shows general tubulin staining, middle row $(\boldsymbol{d}-\boldsymbol{f})$ shows acetylated tubulin stainings, and bottom row $(\boldsymbol{g}-\boldsymbol{i})$ show merged images. Note that microtubule breakpoints in katanin overexpressors have flanking positive acetylated tubulin signals, whereas those of spastin overexpressors do not. Scale bar, $2 \mu \mathrm{m}$. $j$ shows quantification of the experiments by counting discernable breaks. Also included on the graph, as a control, is quantification of randomly selected spots on microtubules (MT) in the same vicinity of control cells, showing frequency of spots being flanked by acetylated regions. In the case of katanin overexpression, there is a significant difference in the frequency of microtubule breaks surrounded by acetylated microtubule signals (Student's $t$ test, ${ }^{*} p<0.01$ ). In the case of spastin overexpression, the difference is not significantly greater could not find consistent significant differences in axons in the same sets of comparisons at the same significance level (i.e., $p<0.01)$. Also, we could not find significant differences $(p>$ 0.01 ) in either axons or dendrites of spastin expressors in the same sets of comparisons as above.

Together, these results indicate a very similar response with the dendrites to what was observed with the fibroblasts, specifically, that 

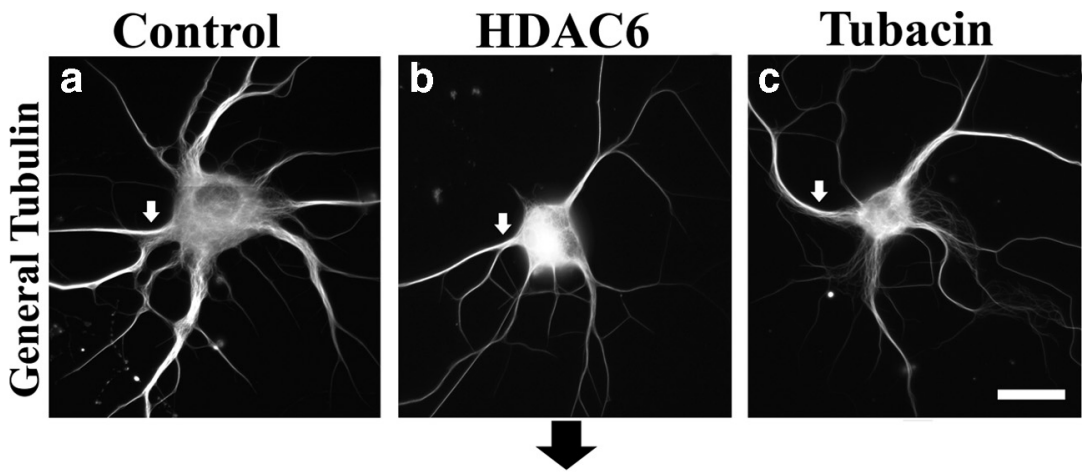

\section{4 hours katanin/spastin expression}

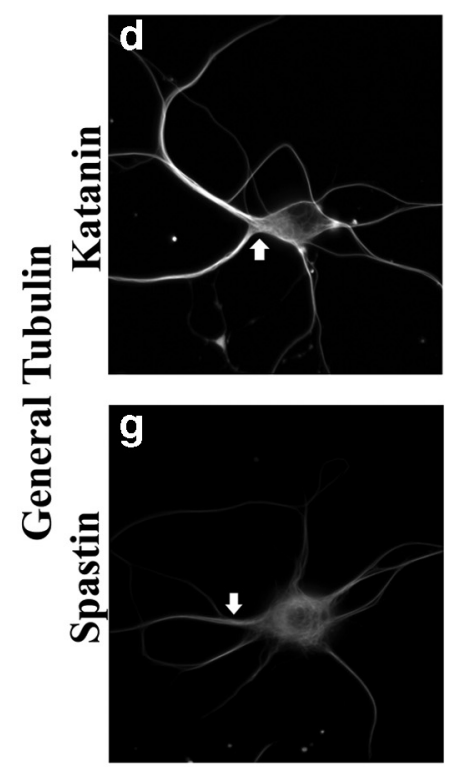

Axons

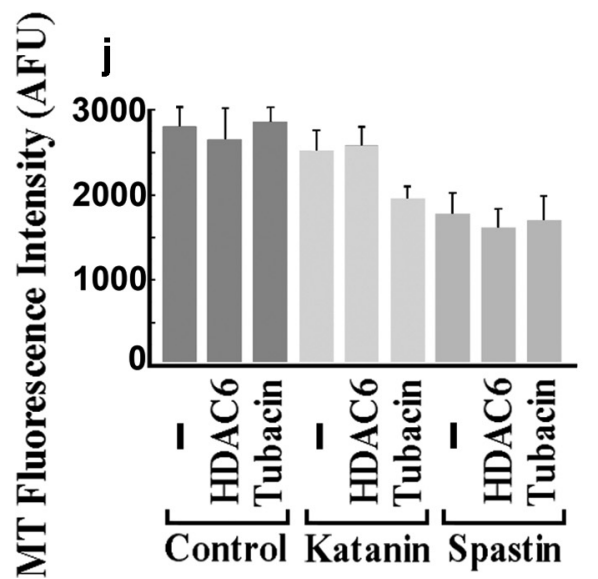

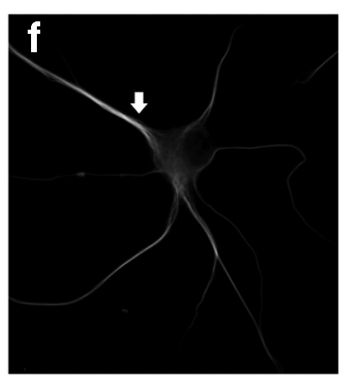
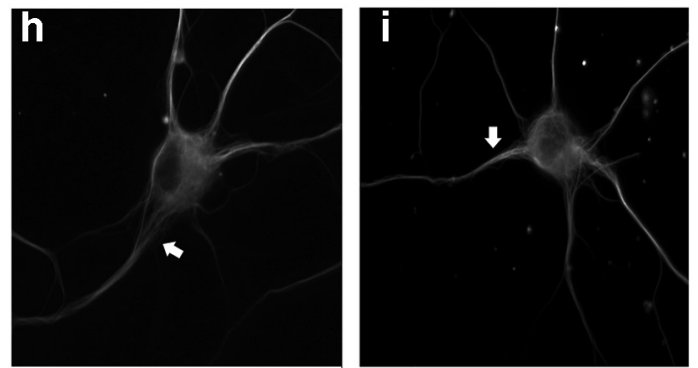

Dendrites

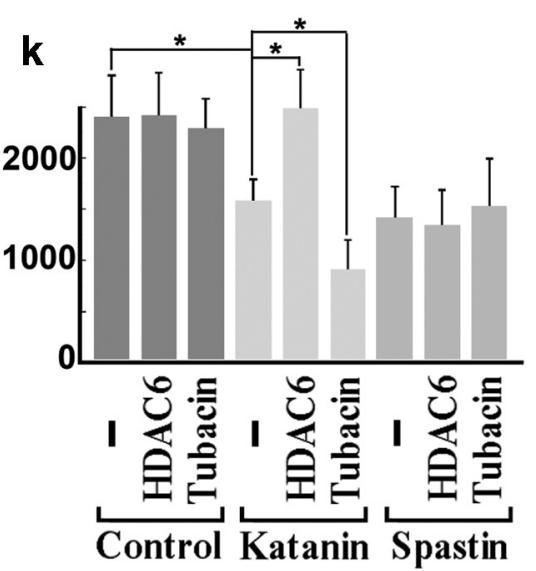

Figure 5. Effects of altered microtubule acetylation on microtubule sensitivity to katanin in neurons. Hippocampal neurons under conditions of HDAC6 overexpression or tubacin treatment were tested for their microtubule sensitivity to overexpressed katanin or spastin. Top row shows control ( $\boldsymbol{a}), \mathrm{HDAC} 6$ overexpression alone $(\boldsymbol{b})$, and tubacin treatment alone (c). Middle row shows katanin overexpressors under the conditions corresponding to the top row. Bottom row shows the spastin overexpressors. Arrows show axons identified by their detailed morphologies as observed with the microscope. All images show general tubulin staining. In katanin overexpression alone (d), significant reduction in microtubule levels was observed in dendrites but not axons. HDAC6 overexpression reduced the sensitivity of microtubules in dendrites $(\boldsymbol{e})$, whereas tubacin treatment enhanced the sensitivity $(\boldsymbol{f})$. Axonal microtubules (MT) did not show changes in sensitivity to overexpressed katanin in the acetylation-dependent manner observed in the case of dendritic microtubules. Spastin overexpressors showed no changes in sensitivity of the microtubules as a result of experimental manipulation of microtubule acetylation. Scale bar, $20 \mu \mathrm{m}$. $\boldsymbol{j}, \boldsymbol{k}$, Quantification of microtubule levels in axons and dendrites by assessing the intensity of general tubulin staining. ${ }^{*} p<0.01$ (Student's $t$ test). elevation and diminution of acetylation causes the microtubules to be more and less sensitive to severing by katanin (but not spastin), respectively. In contrast, this was not the case with the axon.

\section{Tau renders acetylation less}

determinative of microtubule sensitivity to katanin

Tau is generally considered to be an axonal protein (at least the dephosphorylated variant recognized by the Tau-1 antibody) (Bradke and Dotti, 2000) but is not completely absent from dendrites (Kosik and Finch, 1987). In a recent study, we demonstrated that the stronger resistance to katanin of axonal microtubules compared with dendritic microtubules is a result of the presence of tau on the axonal microtubules (Qiang et al., 2006). In this study, when tau was experimentally depleted from the axon, the microtubules became equally as sensitive to katanin overexpression as microtubules in dendrites, immature processes, and the cell body. Based on this, we wondered whether the binding of tau to the microtubules renders the status of their acetylation less influential in determining their sensitivity to katanin. To test this hypothesis, we overexpressed tau in RFL-6 cells (Fig. $6 A a-A f)$, either alone or in conjunction with HDAC6 overexpression or treatment with tubacin. Cells expressing tau alone displayed dense bundles of microtubules (Fig. 6Aa,Ad), and this was also true of tau-overexpressing cells with the two different acetylation manipulations (Fig. $6 A b, A c, A e, A f)$. The HDAC6 was successful in lowering microtubule acetylation, and the tubacin treatment was successful in elevating acetylation in the tauoverexpressing cells (Fig. 6Ae,Af). This was important to confirm because some studies have suggested that tau can interact with HDAC6 (Ding et al., 2008) and inhibit its activity (Perez et al., 2009). We examined whether there were any responses in microtubule sensitivity to altered acetylation in tau-overexpressing RFL-6 cells (Fig. 6B). Katanin overexpressors showed no reduction in microtubules when they coexpressed tau (no microtubule loss in tau and medium katanin coexpressing cells compared with 39\% loss in medium katanin alone-expressing cells), which was consistent with our previous report. The manipulation of acetylation no longer changed the sensitivity (Fig. 6Bf-Bi). Quantitatively, in medium katanin expressors with tau coexpression, only slight reduction or increase in microtubule mass (compared with tau alone) 
A
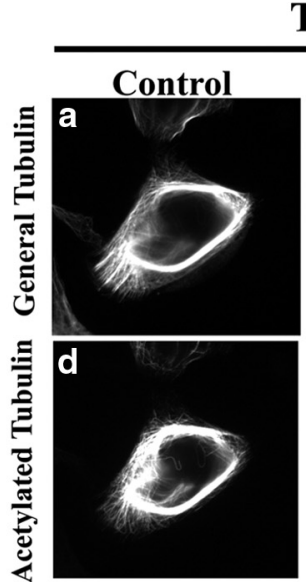

Tau overexpression
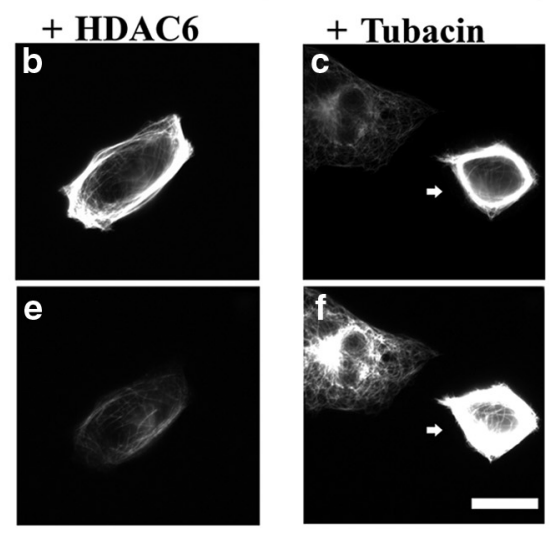

B

Control

Middle level GFP-P60 Katanin expression
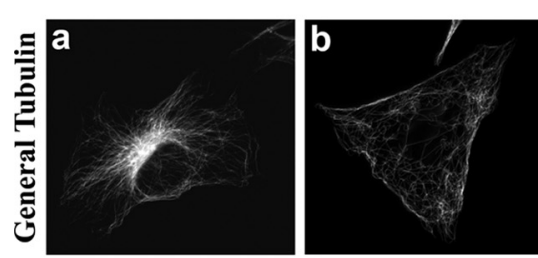

+ HDAC6
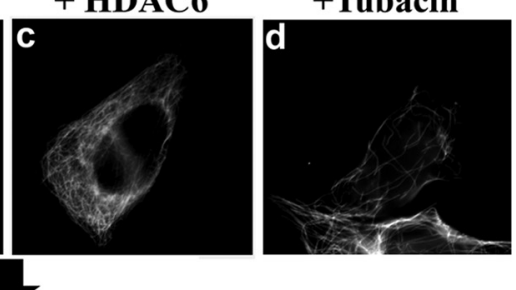

Tau overexpression
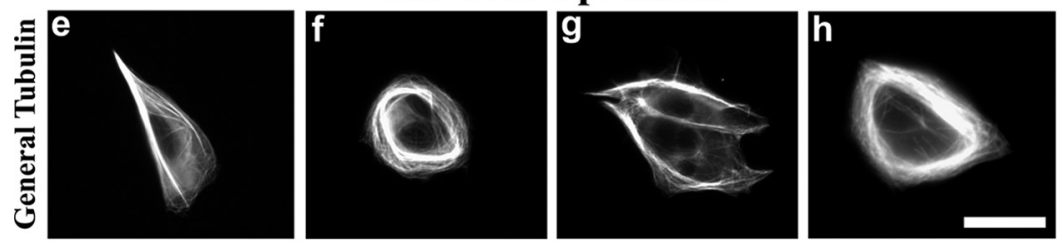

i

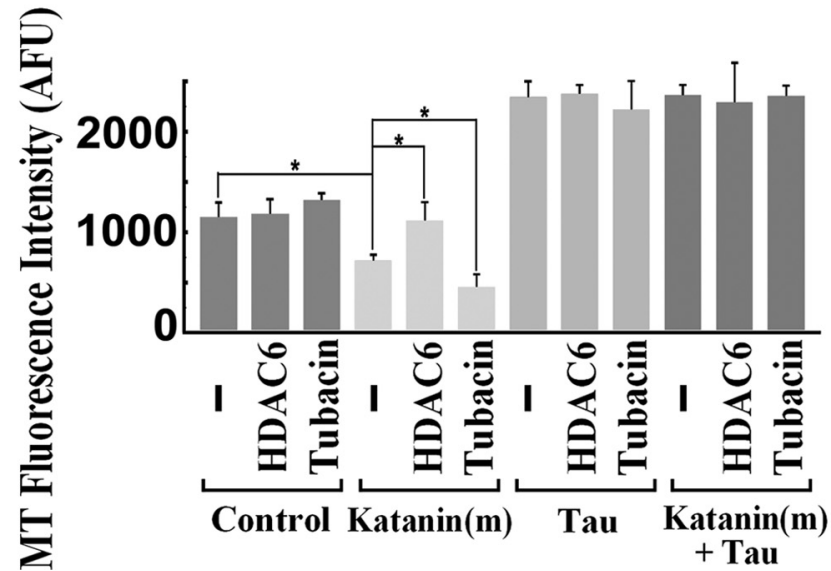

Figure 6. Effects of tau under altered microtubule acetylation on katanin-mediated microtubule severing in RFL-6 cells. $A$, Cells were transfected with tau plasmids under conditions of HDAC6 overexpression or tubacin treatment, fixed, and then immunostained for general tubulin or acetylated tubulin. Left column $(\boldsymbol{a}, \boldsymbol{d})$ shows the control tau overexpressor, middle column $(\boldsymbol{b}, \boldsymbol{e})$ shows tau and HDAC6 coexpressor, and right column (arrows in $\boldsymbol{c}, \boldsymbol{f}$ ) shows the tau overexpressor under tubacin treatment. Top row $(\boldsymbol{a}-\boldsymbol{c})$ shows general tubulin staining, and bottom row $(\boldsymbol{d}-\boldsymbol{f})$ shows acetylated tubulin staining. Tau overexpression caused robust microtubule bundling that was accompanied by enhancement in microtubule acetylation. Note that HDAC6 overexpression and tubacin treatment were effective in reduction or enhancement of microtubule acetylation, respectively. Scale bar, $20 \mu \mathrm{m}$. $\boldsymbol{B}$, Top row $(\boldsymbol{a}-\boldsymbol{d})$ shows cells without tau overexpression, and bottom row $(\boldsymbol{e}-\boldsymbol{h})$ shows cells with tau overexpression. All images show general tubulin staining. Controls $(\boldsymbol{a}, \boldsymbol{e})$ show cells without katanin overexpression. Middle-level katanin overexpression $(\boldsymbol{b}, \boldsymbol{f})$. Middle-level katanin-overexpressing cells with HDAC6 coexpression $(\boldsymbol{c}, \boldsymbol{g})$. Middle-level katanin-overexpressing cells under tubacin treatment $(\boldsymbol{d}, \boldsymbol{h})$. Note that tau-overexpressing cells show characteristic microtubule bundle formation in the absence of katanin overexpression (e), and the microtubule array was almost unchanged in the presence of katanin overexpression $(\boldsymbol{f})$. In the absence of tau overexpression, HDAC6 overexpression resulted in microtubules with less sensitivity to katanin overexpression (c), whereas tubacin treatment had the opposite effect $(\boldsymbol{d})$. However, in the presence of tau overexpression, there are no such changes were observed when they were treated with tubacin or HDAC6, respectively, and these reductions proved to be statistically insignificant $(p$ values $=0.56$ and 0.88 , respectively) ( $n=3,25$ cells were analyzed for each condition). These observations are consistent with the conclusion that the strong protection against katanin afforded by tau overrides the influence of microtubule acetylation observed in the absence of tau.

Finally, we tested whether the sensitivity of ectopically tau-expressing dendrites was changed in response to altered acetylation. For this purpose, we manipulated neurons under tau overexpression and immunostained them for general tubulin and acetylated tubulin (Fig. 7). Consistent with the effects we observed in dendrites in Figure 5, we indeed observed significant effects of our tools with regard to the acetylation levels in all three compartments (Fig. 7a-f). In tau-alone overexpressing neurons, in which we confirmed efficient translocation of expressed tau into dendrites (data not shown), we observed significant changes in the organization of microtubules (Fig. $7 g$ ), including tighter microtubule bundling than that in controls. Tau overexpression induced more acetylation in both axons and dendrites compared with controls (Fig. $7 d, j$ ), but even so, the tools used to alter microtubule acetylation still had demonstrable effects (Fig. $7 k, l$ ).

Figure 8 shows quantitative experiments in which we experimentally manipulated microtubule acetylation in control neurons and neurons overexpressing tau. Compared with acetylation-dependent changes in sensitivity of dendrites in the absence of tau expression (Fig. $8 a-d$ ), we no longer detected any changes in sensitivities that depended on acetylation when tau was overexpressed (Fig. $8 e-h, j$ ). Although we found slight increase in the amount of microtubules in tau expressors, there were also no changes in sensitivities in axons (Fig. 8e-i). Quantitatively, the amount of axonal microtubule in respective conditions are as follows: control (2694 \pm 275$)$, HDAC6 (2610 \pm 97$)$, tubacin $(2507 \pm 179)$, tau $(3283 \pm 324)$, tau + HDAC6 (3447 \pm 162$)$, tau + tubacin $(3424 \pm 171)$, katanin $(2483 \pm 396)$, katanin + HDAC6 (2621 \pm 267$)$, katanin + tubacin $(2249 \pm 263)$, tau + katanin

in sensitivity to katanin $(\boldsymbol{g}, \boldsymbol{h})$. Scale bar, $20 \mu \mathrm{m}$. $\boldsymbol{i}$, Quantification of microtubule (MT) levels by general tubulin staining intensity. ${ }^{*} p<0.01$ (Student's $t$ test). 

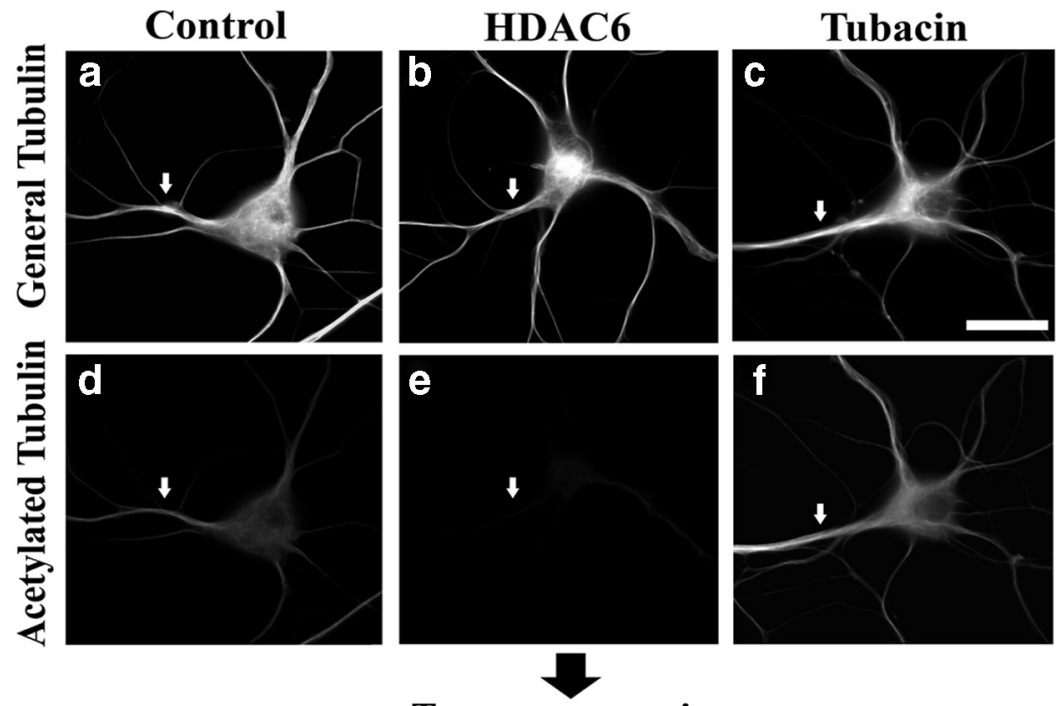

Tau overexpression
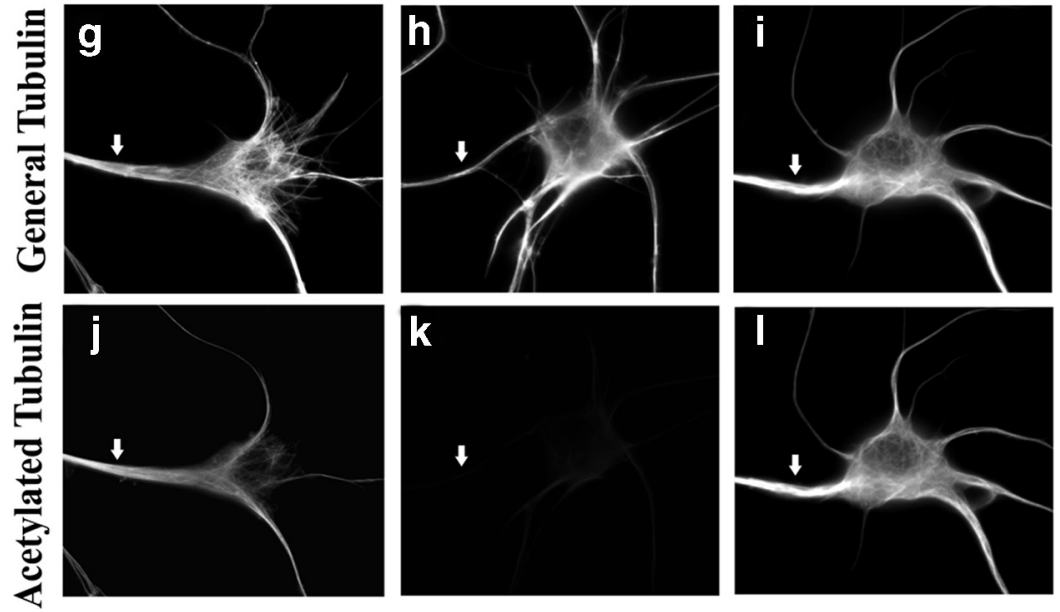

Figure 7. Effects of HDAC6 and tubacin on microtubule acetylation in tau-overexpressing neurons. Neurons were transfected with tau plasmids under conditions of HDAC6 overexpression or tubacin treatment, fixed, and then immunostained for general tubulin or acetylated tubulin. Left column shows control cells with tau overexpression $(\boldsymbol{g}, \boldsymbol{j})$ or without $(\boldsymbol{a}, \boldsymbol{d})$, middle column shows HDAC6-overexpressing cells with tau overexpression $(\boldsymbol{h}, \boldsymbol{k})$ or without $(\boldsymbol{b}, \boldsymbol{e})$, and right column shows tubacin-treated cells with tau overexpression $(\boldsymbol{i}, \boldsymbol{I})$ or without $(\boldsymbol{c}, \boldsymbol{f})$. General tubulin staining $(\boldsymbol{a}-\boldsymbol{c}, \boldsymbol{g}-\boldsymbol{i})$. Acetylated tubulin staining $(\boldsymbol{d}-\boldsymbol{f}, \boldsymbol{j}-\boldsymbol{I})$. Without tau overexpression, HDAC6 overexpression reduced the acetylation of microtubules $(\boldsymbol{e})$, whereas tubacin enhanced it $(\boldsymbol{f})$. Compared with controls $(\boldsymbol{d})$, tau-overexpressing cells show enhanced acetylation of microtubules $(\boldsymbol{j})$. HDAC6 overexpression reduced the acetylation of microtubules $(\boldsymbol{k})$, and tubacin enhanced it even under tau overexpression $(\boldsymbol{I})$. Arrows show axons. Scale bar, $20 \mu \mathrm{m}$.

\section{Discussion}

Microtubule severing is an important event that occurs across many cell types, such as plant cells and epithelial cells, and occurs in many cellular microtubule structures, such as the meiotic and mitotic spindles and cilia (Keating et al., 1997; McNally et al., 2006; Stoppin-Mellet et al., 2006; Sharma et al., 2007; Zhang et al., 2007). We are mainly interested in neurons. Over the years, we have reported that microtubules destined for axons and dendrites are nucleated at the centrosome within the cell body of the neuron and then detached from the centrosome so that molecular motor proteins can convey them into axons and dendrites (Yu et al., 1993; Ahmad et al., 1994; Ahmad and Baas, 1995; Sharp et al., 1996). The ongoing severing of microtubules throughout the neuron is important to generate mobility within the microtubule array, because only very short microtubules are mobile (Wang and Brown, 2002). Microtubule severing is particularly important for growth cone shape and motility (Dent et al., 1999) as well as for the formation of axonal branches (Yu et al., 1994, 2008; Dent et al., 1999; Riano et al., 2009; Qiang et al., 2010). The question arises as to how microtubules in these locales are targeted for a higher frequency of severing than occurs generally along the axonal shaft.

It is notable that the total levels of katanin and spastin in the neuron are very high, exceeding the concentration needed to fully sever purified microtubules into subunits in vitro (Solowska et al., 2008). Thus, a major challenge for the neuron is not only to augment severing in certain locales but also to protect microtubules in other locales from too much severing. We suspect that there are a number of factors that determine when and where the severing of microtubules is most active, including the levels and distribution of the severing proteins themselves (Yu et al.,

$(3272 \pm 416)$, tau + katanin + HDAC6 $(3346 \pm 223)$, and tau + katanin + tubacin $(3467 \pm 137)$ as indicated by [mean microtubule mass (AFU) $\pm \mathrm{SD}$ ] formula $(n=3,25$ cells were analyzed for each condition). Dendritic microtubule in respective conditions are as follows: control $(2152 \pm 185)$, HDAC6 $(2142 \pm 361)$, tubacin $(2064 \pm 268)$, tau $(2702 \pm 421)$, tau + HDAC6 (2851 \pm $462)$, tau + tubacin $(2982 \pm 342)$, katanin $(1308 \pm 261)$, katanin + HDAC6 (2130 \pm 349$)$, katanin + tubacin $(835 \pm 105)$, tau + katanin $(2860 \pm 427)$, tau + katanin + HDAC6 (2822 \pm 556$)$, and tau + katanin + tubacin $(2889 \pm 281)$. In both axons and dendrites, when katanin + tau is compared with katanin + tau + HDAC6 or katanin + tau + tubacin, there are no significant differences $(p>0.01)$ in their microtubule levels.

These data indicate that the sensitivity of microtubules in dendrites to katanin is no longer affected by alterations in acetylation status in the presence of tau.
2005,2008 ), the presence of cofactors such as p80-katanin (Yu et al., 2005), and the composition of microtubule-associated proteins that decorate the microtubule (Baas and Qiang, 2005). Such proteins, especially tau and its family members, strongly suppress the severing of the microtubule by katanin and to a lesser extent by spastin (Qiang et al., 2006; Yu et al., 2008). As such, one potential mechanism for targeting the severing of microtubules is to locally detach such protective proteins from the microtubule in the vicinity in which severing is desirable.

The main purpose of microtubule severing is probably not to depolymerize microtubules but rather to transform smaller numbers of long microtubules into higher numbers of short microtubules. For this reason, it makes sense for cells to target the more stable microtubules for severing so that the resulting short microtubules do not simply depolymerize. Severing of stable mi- 
crotubules would increase the number of microtubules, the number of free microtubule ends, and also potentially render the newly cut microtubules short enough to be actively transported by molecular motor proteins (Baas et al., 2006; RollMecak and Vale, 2006). In this regard, posttranslational modifications of tubulin may be an attractive means by which severing events are targeted to the more stable microtubules, because these modifications are known to accumulate on the most stable microtubules within cells (Schulze et al., 1987; Baas et al., 1991; Bulinski and Gundersen, 1991). Already, it is known that modifications such as acetylation and detyrosination are important for binding of certain motor proteins (Reed et al., 2006; Konishi and Setou, 2009), and hence it would not be surprising if the same were true of severing proteins.

The idea that posttranslational tubulin modifications might be a factor in regulating sensitivity to katanin was originally proposed by Sharma et al. (2007) on the basis of observations on Tetrahymena. In these studies, it was observed that kataninnull mutants displayed dramatic elevations in acetylated and polyglutamylated tubulin (Sharma et al., 2007). On this basis, it was proposed that microtubules rich in these modifications might be the favored target of katanin-induced severing. Additional support for this view was provided in more recent studies on katanin-inhibited cells in culture, which also displayed elevations in microtubule acetylation (Sudo and Maru, 2008). The results reported in our studies more directly demonstrate that acetylation is a significant factor in the sensitivity of a microtubule to katanin. If microtubule acetylation is experimentally elevated or diminished in fibroblasts, the microtubules become more or less sensitive to katanin, respectively. Moreover, observation of recent breaks in microtubules indicates a strong preference for these breaks to be made by katanin in richly acetylated regions of microtubules rather than poorly acetylated regions. Notably, the same does not appear to be true of spastin, because experimental manipulations of acetylation appear to have no effect on the efficiency of spastinmediated microtubule severing, nor does spastin appear to make breaks preferentially in regions of microtubules that are more acetylated (Sharma et al., 2007, wherein no accumulation of acetylated tubulin was observed with spastin nulls). Our studies do not preclude the possibility that other tubulin modifications may play important roles as well in regulating the sensitivity of microtubules to katanin and/or spastin, but our taxol/HDAC6 results (Fig. 2) point to a particularly central role for acetylation in the case of katanin.

This is not the first instance in which we have observed differences in the properties of the two severing proteins. In fact, based on these differences, we have proposed that axonal branches can be produced through either a katanin-based severing of microtu-
Katanin expression

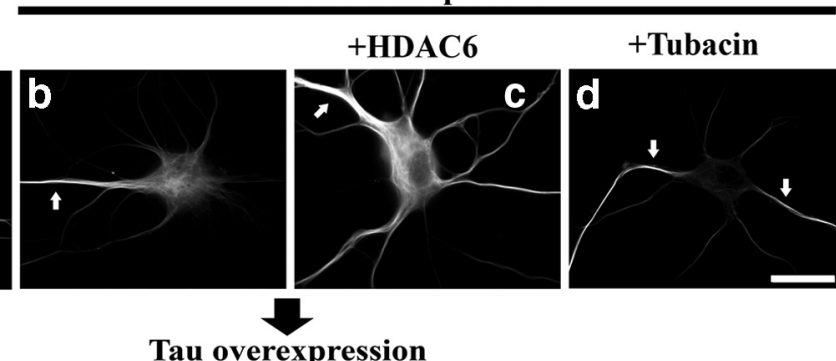

Tau overexpression
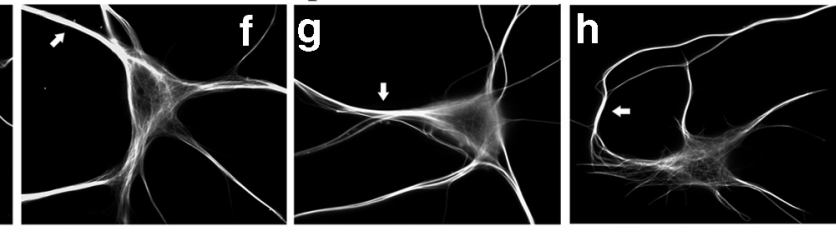

Axons

Dendrites

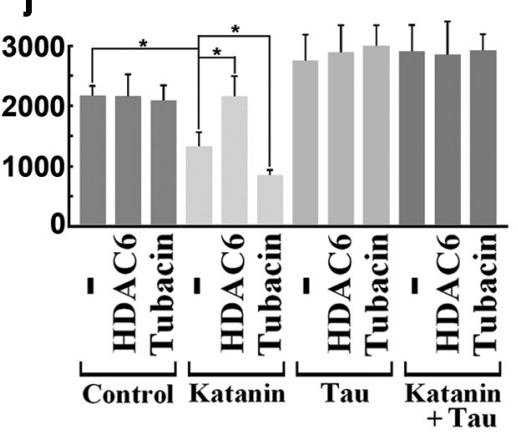

Figure 8. Effects of tau overexpression on microtubule sensitivity to katanin of dendritic microtubules. Neurons were transfected with tau expression plasmids under conditions of HDAC6 overexpression or tubacin treatment and tested for their microtuexpression, and bottom row $(\boldsymbol{e}-\boldsymbol{h})$ shows cells with tau tubacin treatment (h). Arrows show axons. Scale bar, $20 \mu \mathrm{m} . \boldsymbol{i}, \boldsymbol{j}$, Quantification of microtubule (MT) levels in axons and dendrites by assessing the intensity of general tubulin signals. ${ }^{*} p<0.01$ (Student's $t$ test).

bules or a spastin-based severing of microtubules ( $\mathrm{Yu}$ et al., 2008). In the case of katanin, the severing of microtubules at the appropriate sites is activated by the dissociation of tau and other protective proteins from the microtubule lattice. In the case of spastin, it is simply the focal accumulation of spastin that elicits sufficient severing at the site of branch formation. In neurons, using immunoelectron microscopy, we have documented dramatic heterogeneity in the levels of acetylation along the length of individual microtubules, with the highest levels existing in discrete patches within the more stable domains of the polymer (Baas et al., 1991). Such patches, similar to those observed in the present studies on fibroblasts, may represent the most sensitive sites for potential severing by katanin.

In dendrites, the microtubules are less acetylated than in axons but still display regions of acetylation (Baas et al., 1991). In addition, it appears that the normal complement of MAPs on dendritic microtubules provides far less protection against katanin than the normal MAP complement of axonal microtubules. The reasons for this remain murky because dendrites contain MAP2 as well as some tau, both of which can protect microtubules against katanin (Qiang et al., 2006). Perhaps the difference 
lies in the amounts of these proteins decorating the microtubules in dendrites compared with axons, because overexpression of tau affords protection of dendritic microtubules against katanin. In any event, potential regions of enhanced acetylation on dendritic microtubules may be quite important for targeting severing of microtubules during dendritic differentiation. In support of this, it has been reported that acetylation of microtubules in dendrites is key for them to undergo appropriate branching (Ohkawa et al., 2008). For microtubule acetylation to play an important role in axonal branching, mechanisms would presumably have to exist to coordinate the acetylation status of the microtubule with the binding of tau in distinct locales of the axon.

The most perplexing issue that arises from our studies is exactly how a modification of $\alpha$ tubulin that occurs on the luminal face of the polymer could alter the sensitivity of the microtubule to katanin. One possibility is that the acetylation status alters the lattice of the polymer in such a way as to influence the efficiency with which different proteins interact with it. If this is correct, it remains to be resolved whether the affinity of the lattice for katanin is directly affected or whether the effect is indirect, through the association of other proteins that are sensitive to subtle alterations in the lattice of the microtubule (such as Hsp90) (Giustiniani et al., 2009). There is already evidence that so-called "lattice flaws" in the microtubule are especially targeted for severing by katanin (Odde et al., 1999). Another possibility relates to the manner by which the severing proteins break the microtubule. Specifically, a domain of the severing protein reaches into the microtubule, through the lattice, and yanks out a subunit, thus causing the microtubule to break (Roll-Mecak and McNally, 2010). In this sense, the fact that acetylation occurs on the luminal face of the microtubule may actually provide the ideal location to influence the efficacy of the severing event. Interestingly, tau is thought to bind to the inside wall of the microtubules (Kar et al., 2003), which is consistent with the possibility that the sensitivity of the microtubule to katanin is regulated mainly by events on the luminar face of the microtubule.

\section{References}

Ahmad FJ, Baas PW (1995) Microtubules released from the neuronal centrosome are transported into the axon. J Cell Sci 108:2761-2769.

Ahmad FJ, Joshi HC, Centonze VE, Baas PW (1994) Inhibition of microtubule nucleation at the neuronal centrosome compromises axon growth. Neuron 12:271-280.

Baas PW, Qiang L (2005) Neuronal microtubules: when the MAP is the roadblock. Trends Cell Biol 15:183-187.

Baas PW, Slaughter T, Brown A, Black MM (1991) Microtubule dynamics in axons and dendrites. J Neurosci Res 30:134-153.

Baas PW, Vidya Nadar C, Myers KA (2006) Axonal transport of microtubules: the long and short of it. Traffic 7:490-498.

Bradke F, Dotti CG (2000) Differentiated neurons retain the capacity to generate axons from dendrites. Curr Biol 10:1467-1470.

Bulinski JC, Gundersen GG (1991) Stabilization of post-translational modification of microtubules during cellular morphogenesis. Bioessays 13:285-293.

Cambray-Deakin MA, Burgoyne RD (1987) Posttranslational modifications of alpha-tubulin: acetylated and detyrosinated forms in axons of rat cerebellum. J Cell Biol 104:1569-1574.

Chang J, Baloh RH, Milbrandt J (2009) The NIMA-family kinase Nek3 regulates microtubule acetylation in neurons. J Cell Sci 122:2274-2282.

Dent EW, Callaway JL, Szebenyi G, Baas PW, Kalil K (1999) Reorganization and movement of microtubules in axonal growth cones and developing interstitial branches. J Neurosci 19:8894-8908.

Ding H, Dolan PJ, Johnson GV (2008) Histone deacetylase 6 interacts with the microtubule-associated protein tau. J Neurochem 106:2119-2130.

Giustiniani J, Daire V, Cantaloube I, Durand G, Poüs C, Perdiz D, Baillet A (2009) Tubulin acetylation favors Hsp90 recruitment to microtubules and stimulates the signaling function of the Hsp90 clients Akt/PKB and p53. Cell Signal 21:529-539.

Gundersen GG, Khawaja S, Bulinski JC (1987) Postpolymerization detyrosination of alpha-tubulin: a mechanism for subcellular differentiation of microtubules. J Cell Biol 105:251-264.

Haggarty SJ, Koeller KM, Wong JC, Grozinger CM, Schreiber SL (2003) Domain-selective small-molecule inhibitor of histone deacetylase 6 (HDAC6)-mediated tubulin deacetylation. Proc Natl Acad Sci U S A 100:4389-4394.

Hammond JW, Cai D, Verhey KJ (2008) Tubulin modifications and their cellular functions. Curr Opin Cell Biol 20:71-76.

Hubbert C, Guardiola A, Shao R, Kawaguchi Y, Ito A, Nixon A, Yoshida M, Wang XF, Yao TP (2002) HDAC6 is a microtubule-associated deacetylase. Nature 417:455-458.

Ikegami K, Heier RL, Taruishi M, Takagi H, Mukai M, Shimma S, Taira S, Hatanaka K, Morone N, Yao I, Campbell PK, Yuasa S, Janke C, Macgregor GR, Setou M (2007) Loss of alpha-tubulin polyglutamylation in ROSA22 mice is associated with abnormal targeting of KIF1A and modulated synaptic function. Proc Natl Acad Sci U S A 104:3213-3218.

Kar S, Fan J, Smith MJ, Goedert M, Amos LA (2003) Repeat motifs of tau bind to the insides of microtubules in the absence of taxol. EMBO J 22:70-77.

Karabay A, Yu W, Solowska JM, Baird DH, Baas PW (2004) Axonal growth is sensitive to the levels of katanin, a protein that severs microtubules. J Neurosci 24:5778-5788.

Keating TJ, Peloquin JG, Rodionov VI, Momcilovic D, Borisy GG (1997) Microtubule release from the centrosome. Proc Natl Acad Sci U S A 94:5078-5083.

Konishi Y, Setou M (2009) Tubulin tyrosination navigates the kinesin-1 motor domain to axons. Nat Neurosci 12:559-567.

Kosik KS, Finch EA (1987) MAP2 and tau segregate into dendritic and axonal domains after the elaboration of morphologically distinct neurites: an immunocytochemical study of cultured rat cerebrum. J Neurosci 7:3142-3153.

Matsuyama A, Shimazu T, Sumida Y, Saito A, Yoshimatsu Y, SeigneurinBerny D, Osada H, Komatsu Y, Nishino N, Khochbin S, Horinouchi S, Yoshida M (2002) In vivo destabilization of dynamic microtubules by HDAC6-mediated deacetylation. EMBO J 21:6820-6831.

McNally K, Audhya A, Oegema K, McNally FJ (2006) Katanin controls mitotic and meiotic spindle length. J Cell Biol 175:881-891.

Odde DJ, Ma L, Briggs AH, DeMarco A, Kirschner MW (1999) Microtubule bending and breaking in living fibroblast cells. J Cell Sci 112:3283-3288.

Ohkawa N, Sugisaki S, Tokunaga E, Fujitani K, Hayasaka T, Setou M, Inokuchi K (2008) N-acetyltransferase ARD1-NAT1 regulates neuronal dendritic development. Genes Cells 13:1171-1183.

Perez M, Santa-Maria I, Gomez de Barreda E, Zhu X, Cuadros R, Cabrero JR, Sanchez-Madrid F, Dawson HN, Vitek MP, Perry G, Smith MA, Avila J (2009) Tau: an inhibitor of deacetylase HDAC6 function. J Neurochem 109:1756-1766.

Piperno G, LeDizet M, Chang XJ (1987) Microtubules containing acetylated alpha-tubulin in mammalian cells in culture. J Cell Biol 104:289-302.

Pugacheva EN, Jablonski SA, Hartman TR, Henske EP, Golemis EA (2007) HEF1-dependent Aurora A activation induces disassembly of the primary cilium. Cell 129:1351-1363.

Qiang L, Yu W, Andreadis A, Luo M, Baas PW (2006) Tau protects microtubules in the axon from severing by katanin. J Neurosci 26:3120-3129.

Qiang L, Yu W, Liu M, Solowska JM, Baas PW (2010) Basic fibroblast growth factor elicits formation of interstitial axonal branches via enhanced severing of microtubules. Mol Biol Cell 21:334-344.

Redeker V, Levilliers N, Vinolo E, Rossier J, Jaillard D, Burnette D, Gaertig J, Bré MH (2005) Mutations of tubulin glycylation sites reveal cross-talk between the $\mathrm{C}$ termini of alpha- and beta-tubulin and affect the ciliary matrix in Tetrahymena. J Biol Chem 280:596-606.

Reed NA, Cai D, Blasius TL, Jih GT, Meyhofer E, Gaertig J, Verhey KJ (2006) Microtubule acetylation promotes kinesin-1 binding and transport. Curr Biol 16:2166-2172.

Riano E, Martignoni M, Mancuso G, Cartelli D, Crippa F, Toldo I, Siciliano G, Di Bella D, Taroni F, Bassi MT, Cappelletti G, Rugarli EI (2009) Pleiotropic effects of spastin on neurite growth depending on expression levels. J Neurochem 108:1277-1288.

Roll-Mecak A, McNally FJ (2010) Microtubule-severing enzymes. Curr Opin Cell Biol 22:96-103. 
Roll-Mecak A, Vale RD (2006) Making more microtubules by severing: a common theme of noncentrosomal microtubule arrays? J Cell Biol 175:849-851.

Schulze E, Asai DJ, Bulinski JC, Kirschner M (1987) Posttranslational modification and microtubule stability. J Cell Biol 105:2167-2177.

Sharma N, Bryant J, Wloga D, Donaldson R, Davis RC, Jerka-Dziadosz M, Gaertig J (2007) Katanin regulates dynamics of microtubules and biogenesis of motile cilia. J Cell Biol 178:1065-1079.

Sharp DJ, Kuriyama R, Baas PW (1996) Expression of a kinesin-related motor protein induces Sf9 cells to form dendrite-like processes with nonuniform microtubule polarity orientation. J Neurosci 16:43704375.

Solowska JM, Morfini G, Falnikar A, Himes BT, Brady ST, Huang D, Baas PW (2008) Quantitative and functional analyses of spastin in the nervous system: implications for hereditary spastic paraplegia. J Neurosci 28:2147-2157.

Stoppin-Mellet V, Gaillard J, Vantard M (2006) Katanin's severing activity favors bundling of cortical microtubules in plants. Plant J 46:1009-1017.

Sudo H, Maru Y (2008) LAPSER1/LZTS2: a pluripotent tumor suppressor linked to the inhibition of katanin-mediated microtubule severing. Hum Mol Genet 17:2524-2540.
Wang L, Brown A (2002) Rapid movement of microtubules in axons. Curr Biol 12:1496-1501.

Yu W, Baas PW (1994) Changes in microtubule number and length during axon differentiation. J Neurosci 14:2818-2829.

Yu W, Centonze VE, Ahmad FJ, Baas PW (1993) Microtubule nucleation and release from the neuronal centrosome. J Cell Biol 122:349-359.

Yu W, Solowska JM, Qiang L, Karabay A, Baird D, Baas PW (2005) Regulation of microtubule severing by katanin subunits during neuronal development. J Neurosci 25:5573-5583.

Yu W, Qiang L, Solowska JM, Karabay A, Korulu S, Baas PW (2008) The microtubule-severing proteins spastin and katanin participate differently in the formation of axonal branches. Mol Biol Cell 19:1485-1498.

Zhang D, Rogers GC, Buster DW, Sharp DJ (2007) Three microtubule severing enzymes contribute to the "Pacman-flux" machinery that moves chromosomes. J Cell Biol 177:231-242.

Zhao Z, Xu H, Gong W (2010) Histone deacetylase 6 (HDAC6) is an independent deacetylase for alpha-tubulin. Protein Pept Lett 17:555-558.

Zilberman Y, Ballestrem C, Carramusa L, Mazitschek R, Khochbin S, Bershadsky A (2009) Regulation of microtubule dynamics by inhibition of the tubulin deacetylase HDAC6. J Cell Sci 122:3531-3541. 